\title{
шा
}

VIsen

\section{Noninvasive, in vivo quantification of asthma severity using fluorescence molecular tomography}
VisEn Medical has developed FMT ${ }^{\mathrm{TM}} 2500$, a quantitative fluorescence molecular tomographic system useful for rapid, noninvasive measurement of disease biology in living animals. This imaging technology, paired with one of VisEn's protease-activatable agents, ProSense 680 , has enabled the quantification of disease-related protease activity in the mouse ovalbumin-induced model of asthma.

\begin{abstract}
Asthma is an inflammatory disease process characterized by reversible airway obstruction and airway hyperresponsiveness. This disease process is driven by activated T lymphocytes and eosinophils that are recruited to the lung upon inhalation of triggering allergens. These cells release inflammatory mediators, activate mast cells and epithelial cells and stimulate mucus secretion, ultimately leading to airway obstruction. The incidence and severity of asthma is increasing worldwide, elevating the need for clinically relevant in vivo animal models that can be used to improve the understanding of asthma biology and the development of effective therapeutics. Here we illustrate the use of VisEn's FMT 2500 quantitative tomography in combination with ProSense 680, a near-infrared, protease-activatable fluorescence agent, for the noninvasive in vivo imaging and quantification of pulmonary inflammation.
\end{abstract}

Noninvasive quantitative tomography of mouse asthma Asthma can be induced in female BALB/c mice by provoking specific immunity to ovalbumin in the lung ${ }^{1}$. To do this, we immunized 6- to 8 -week-old BALB/c mice on day 0 and day 14 by intraperitoneal injections of $50 \mu \mathrm{g}$ of ovalbumin combined with $2 \mathrm{~g}$ of aluminum hydroxide as an adjuvant to enhance the allergic response. Between day 21 and day 24, mice received daily intranasal challenges with $100 \mu \mathrm{g}$ of ovalbumin solubilized in PBS (pH 7.4) to focus an ovalbumin-specific allergic response within the airways. The allergic response to ovalbumin induces cytokines and immune factors typical of those in human asthma (for example, interleukin (IL)-4, histamine and IgE), a large influx of eosinophils and changes in airway hyperreactivity. Measurement of these parameters requires surgical procedures (for airway hyperreactivity), assessment of killed mice (for bronchoalveolar lavage (BAL) eosinophil count) and extensive sample handling and preparation (for serum and BAL fluid microplate assays)².

\section{Houari Korideck \& Jeffrey D Peterson}

VisEn Medical, 45 Wiggins Avenue, Bedford, Massachusetts 01730, USA. Correspondence should be addressed to J.D.P. (jpeterson@visenmedical.com).

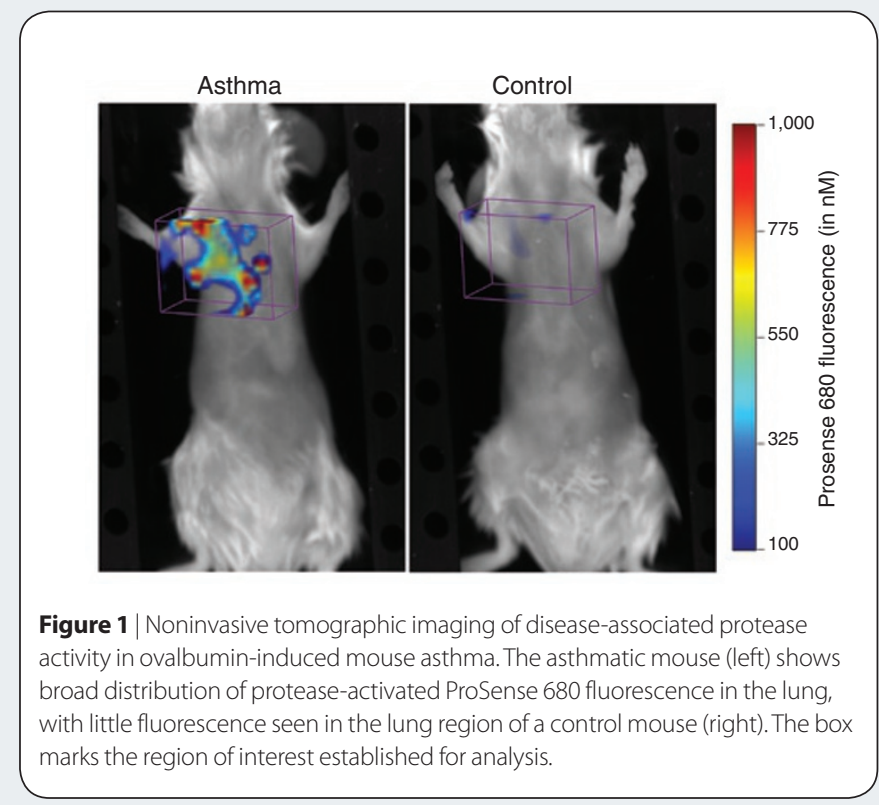

VisEn's fluorescence-based quantitative tomography offers a noninvasive alternative to current invasive and terminal assessments of mouse pulmonary inflammation ${ }^{3}$. At the core of this technology is VisEn's fluorescence molecular tomography system, the FMT 2500. This system uses trans-illumination (that is, passing near-infrared light through the imaging subject) from multiple viewpoints, providing a rich dataset to mathematically model and reconstruct tomographic quantitative distribution of all sources of fluorescence within the subject.

Pairing this technology with one of VisEn's near-infrared fluorescence agents, ProSense 680, allows the accurate detection and quantification of protease activity associated with eosinophils ${ }^{4}$; ProSense 680 is optically silent and fluoresces only when activated by diseaserelated proteases such as cathepsins. The near-infrared fluorochrome allows maximal tissue penetration and minimal absorption by physiological absorbers such as hemoglobin or water. 
a

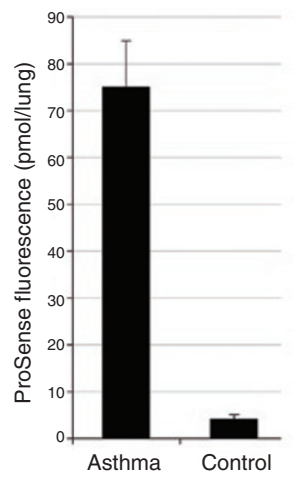

b

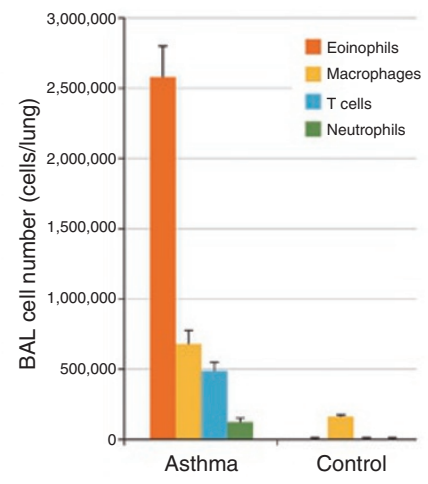

Figure 2 Assessment of asthma severity using VisEn's FMT 2500 quantitative tomography (noninvasive) and bronchoalveolar lavage cell counts (terminal). (a) Quantification of tomographic images shows high amounts of ProSense 680 fluorescence within the lung region of asthmatic mice, with minimal fluorescence measured within that of control mice. Error bars, \pm s.e.m. (b) BAL assessment indicates large numbers of eosinophils infiltrating the lungs of asthmatic mice compared to those of control mice, consistent with in vivo imaging results. The experiment is representative of five separate studies; $n=5$ mice per group. Error bars, \pm s.e.m.

To quantify the inflammation associated with asthma progression, we injected the mice with $5 \mathrm{nmol}$ of Prosense 680 at day 24, 4 hours after the final intranasal administration of ovalbumin, that is, 24 hours before imaging. On the day of imaging, we anesthetized the mice and positioned them in the imaging chamber of the FMT 2500. This system provides both tomographic (for deep-tissue fluorescence) and two-dimensional fluorescence reflectance imaging (for skin fluorescence and assessment of excised organs and tissues). Tomographic imaging of asthmatic mice yielded clear fluorescence signal in lungs of asthmatic mice, but not in control mice that received PBS instead of ovalbumin intranasal challenge (Fig. 1). Analysis of the tomographic imaging data sets measured a highly significant increase in activated ProSense fluorescence in the lung ( $>70$ $\mathrm{pmol} / \mathrm{lung}$ ) as compared to $<5 \mathrm{pmol}$ in the lungs of control mice $(P=0.002$; Fig. 2a). These results correlate well with the eosinophil count from BAL samples (Fig. 2b).

Asthmatic lungs removed from euthanized mice showed widespread fluorescence (Fig. 3a), with minimal fluorescent background evident in control tissue. Measurement of the ratios of fluorescence intensity in lungs of mice with induced asthma yielded signal intensity $>3.5$-fold higher than that in lungs of control mice (Fig. 3b). Notably, the individual fluorescence ratios for each excised lung correlated well with the noninvasive quantification performed by FMT 2500 (Fig. 3c), supporting the contention that tomographic assessment was solely quantifying lung fluorescence. This confirms that the noninvasive tomographic assessment of asthmatic mice can be used to accurately assess deep tissue changes in protease activity associated with asthma, in agreement with BAL eosinophil counts (Fig. 2b).

a

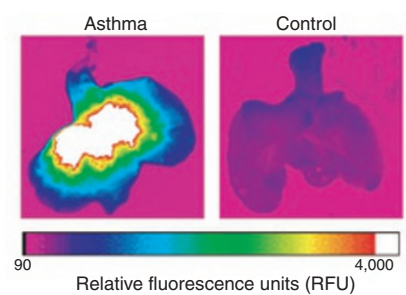

b

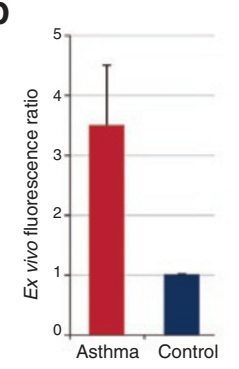

C

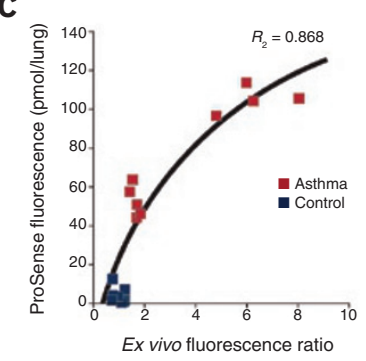

Figure 3 | Ex vivo correlation between noninvasive quantitative tomography and exvivo tissue assessment. (a) Excised lung tissues were assessed for ProSense 680 fluorescence changes, with images revealing significant asthma-related increases in lung fluorescence intensity. (b) Assessment of changes in ex vivo fluorescence ratio (ratio to mean control tissue fluorescence) in lung tissue from mice with induced asthma and from control mice ( $P<0.05$; asthma tissue to control tissue fluorescence ratio \pm s.e.m.). (c) Noninvasive FMT 2500 tomographic quantification correlates well with the signal in the excised lung tissues. The results shown are representative of five separate studies; $n=5$ mice per group.

\section{Conclusion}

These studies demonstrate the utility of FMT 2500 quantitative tomography in combination with a near-infrared, protease-activatable fluorescence agent (ProSense 680), for the noninvasive in vivo imaging and quantification of pulmonary inflammation. After inducing asthma by ovalbumin in BALB/c mice, we effectively imaged ProSense 680 to detect eosinophil protease signal that can be tomographically quantified by VisEn's FMT 2500. Correlation of noninvasive tomographic quantification with invasive assessment of lung inflammation established the accuracy of FMT 2500 quantification of deep tissue inflammation. VisEn's FMT 2500 also offers the potential for repeated noninvasive imaging and multiplex detection of multiple biological activities, allowing researchers to better study the progression and alteration of processes associated with disease progression and therapeutic intervention.

1. Brusselle, G.G. et al. Attenuation of allergic airway inflammation in IL-4 deficient mice. Clin. Exp. Allergy 24, 73-80 (1994).

2. Kim, S.J. et al. Time sequence of airway remodeling in a mouse model of chronic asthma: the relation with airway hyperresponsiveness. J. Korean Med. Sci. 22, 183-191 (2007).

3. Haller, J.L. et al. Visualization of pulmonary inflammation using non-invasive fluorescence molecular imaging. J. Appl. Physiol. 104, 795-802 (2008).

4. Fulkerson, P.C., Fischetti, C.A. \& Rothenberg, M.E. Eosinophils and CCR3 regulate interleukin-13 transgene-induced pulmonary remodeling. Am. J. Pathol. 169 2117-2126 (2006).

This article was submitted to Nature Methods by a commercial organization and has not been peer reviewed. Nature Methods takes no responsibility for the accuracy or otherwise of the information provided. 